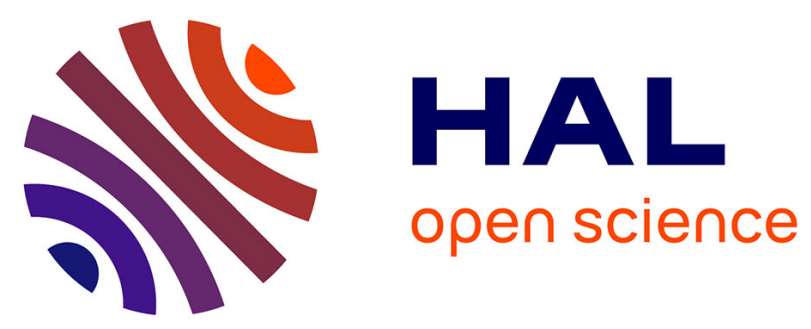

\title{
L'errance chez les sujets déments en institution
}

Priscilla Poitevin

\section{To cite this version:}

Priscilla Poitevin. L'errance chez les sujets déments en institution. NPG: Neurologie - Psychiatrie -

Gériatrie, 2019, 19, pp.73 - 76. 10.1016/j.npg.2018.12.002 . hal-03486352

\section{HAL Id: hal-03486352 \\ https://hal.science/hal-03486352}

Submitted on 21 Dec 2021

HAL is a multi-disciplinary open access archive for the deposit and dissemination of scientific research documents, whether they are published or not. The documents may come from teaching and research institutions in France or abroad, or from public or private research centers.
L'archive ouverte pluridisciplinaire HAL, est destinée au dépôt et à la diffusion de documents scientifiques de niveau recherche, publiés ou non, émanant des établissements d'enseignement et de recherche français ou étrangers, des laboratoires publics ou privés.

\section{다)(1) $(5$}

Distributed under a Creative Commons Attribution - NonCommercial| 4.0 International 


\section{L'ERRANCE CHEZ LES SUJETS DEMENTS EN INSTITUTION}

\section{WANDERING AMONG PEOPLE WITH DEMENTIA IN INSTITUTIONS}

Titre abrégé: L'errance chez le sujet dément

Short title: Wandering among subjects with dementia

Priscilla POITEVIN

Psychologue clinicienne, doctorante en psychopathologie et psychologie clinique au Laboratoire CRPPC (Centre de Recherche en psychopathologie et psychologie clinique) - EA 653 à l'Université Lumière Lyon 2.

20, rue Perdonnet 75010 Paris

pkpoitevin@yahoo.com.br

Tel: 0680765363 


\section{Résumé}

Cet article propose une réflexion sur l'errance, à partir d'un travail d'accompagnement de deux résidentes présentant ce symptôme en institution. Dans un premier temps, nous soulèverons les problématiques conceptuelles concernant la notion de déambulation et leur importance dans le choix du terme «errance». Ensuite, deux vignettes cliniques seront présentées afin d'analyser l'hypothèse selon laquelle cette activité motrice peut être une modalité d'apaisement de l'angoisse, ou encore, un moyen de faire persister la dimension subjective du sujet. Pour finir, nous soulignerons les limites d'une telle recherche et des difficultés rencontrées par le psychologue lors de l'accompagnement des sujets errants.

Mots clés : démence ; psyché-soma ; errance ; institution

\section{Summary}

This article introduces a reflection on wandering/straying among demented subjects based on the cases of two women presenting this symptom. Firstly, we explore the conceptual issues behind the notion of "wandering" and the importance of the choice of the term. We then discuss the two clinical cases, leading us to a hypothesis according to which this activity not only provides a means for these individuals to relieve their anxiety, but could also be seen as a way to express their individuality. Finally, we discuss the limits of this research and the difficulties encountered by the clinician in the accompaniment of wandering or straying subjects.

Keywords : dementia; psyche-soma; wandering; institution 
Dans le quotidien du travail en établissement d'hébergement pour personnes âgées (EHPAD), nous sommes confrontés à des sujets qui nous révèlent le lien indissociable entre le somatique et la psyché. Des corps qui parlent, sans dire un mot, qui s'agitent au point de se laisser tomber, des corps qui sont marqués par une fragilité non seulement liée à l'âge mais aussi à leur dépendance. Et parmi les manifestations corporelles qui se produisent au cours des maladies neurodégénératives, l'errance apparaît désormais comme l'une des raisons principales pour l'admission en institution et l'une des grandes difficultés rencontrées par l'équipe soignante au cours de la prise en charge du résident errant. A partir d'une orientation psychanalytique, il sera proposé de partager une réflexion sur la question de l'errance chez certains sujets dits «déments». Dans un premier temps, il est important de soulever les problématiques conceptuelles concernant la déambulation et d'expliquer le choix du mot «errance ». Ensuite, nous proposons deux vignettes cliniques relevant de la clinique de l'errance. La conclusion présentera les limites concernant l'intervention du psychologue dans l'accompagnement des sujets errants.

\section{De la déambulation à l'errance : problématiques conceptuelles}

De nombreuses études publiées en France et à l'étranger révèlent que la notion de déambulation a évolué depuis quelques décennies, même s’il n’existe pas «de définition consensuelle de ce terme, qui soit appliquée à des situations extrêmement diverses dans leur expression clinique et leur signification »[1]. La complexité du symptôme psychomoteur nécessite la prise en compte de la relation résident/famille/institution et de la situer au croisement des approches médicale, politique, sociale, éthique et psychologique.

L'idée de mettre l'errance à l'épreuve de la psyché implique le rapport singulier du sujet à son corps au travers de l'hypothèse que l'envie d'aller là-bas, d'aller plus loin et même parfois au- 
delà, soit une solution pour lutter contre l'angoisse: l'angoisse de la maladie, de l'enfermement, du sentiment d'inquiétante étrangeté quant à son propre corps, l'angoisse de mort. Si l'angoisse est un affect qui ne trompe pas [2], comme le dit Lacan, elle est indice de vérité pour le sujet confronté à ce réel insupportable de la finitude humaine.

\section{Les cent « pas » de Madame B.}

Madame B, âgée de 79 ans, est arrivée à la résidence au mois de septembre 2016. Son entrée en EHPAD faisait suite tout d'abord à une aggravation de ses troubles cognitifs et comportementaux, mais aussi de l'épuisement de l'aidant principal, en l'occurrence, sa fille, qui avait perdu son mari six mois auparavant, lui-même souffrant d'une démence. Son dossier médical révèle plusieurs AVC survenus deux ans avant le diagnostic de démence vasculaire avancée. Dès ses premiers jours dans l'établissement, Madame B. erre dans les couloirs, entre dans toutes les chambres, surtout la nuit où elle va jusqu'à dormir dans le lit d'autres résidents. Ce qui interroge vite l'équipe sur la nécessité de l'héberger dans une unité protégée. Lors de notre première rencontre, Madame B. est devant la porte qui mène au jardin de la résidence et semble prononcer des syllabes incompréhensibles. Ensuite, elle dit :

«C'est con con con. L'autre c'est moi. Je voulais pas... payer enfin pa pa pa »

Nous lui répétons les trois «pas » prononcés à la fin de sa phrase et invitons à en faire quelques uns avec elle, mais elle part... sans répondre. Il y a de la musique dans la salle à manger, Madame B. balance alors les bras comme une vague et déambule dans un parcours qui va jusqu'à l'accueil de l'établissement, en passant par la grande salle et les couloirs menant aux chambres... Arrivée à l'accueil, elle s'arrête devant un miroir et l'embrasse, puis elle dit : «Ça fait deux » et elle sourit. C'est à partir de cette rencontre avec son altérité que démarre son parcours d'errance. 
L'écholalie de Madame B. se présente régulièrement à la fin de ses phrases, et lorsqu'elle répète le «pas »- ce qui lui arrive assez souvent -, il est difficile de comprendre ce que cela veut dire. Dans le discours, il est fondamental de ne pas prendre les paroles au pied de la lettre, surtout dans la clinique de la démence. La richesse de la langue française nous inspire à envisager trois hypothèses.

Une première interprétation peut nous amener à envisager le «pas » de la négation. Dans ce cas, à quoi dit-elle non? Selon sa fille, Madame B. n'a jamais voulu entrer en EHPAD et a toujours eu un discours négatif concernant ces lieux de vie pour personnes âgées. Son errance serait-elle un acte de refus de se faire objet de l'institution?

Nous pouvons aussi envisager une deuxième possibilité, si on entend le «pas » comme l'action de marcher. Cela relèverait d'une interaction psychodynamique où le corps en mouvement semble être un pur objet pulsionnel cherchant la satisfaction à tout prix. Selon Freud [3], la pulsion est définie comme un concept limite entre le somatique et le psychique. Elle se représente à partir des affects associés. Madame B. nous ferait-elle savoir par son attitude d'apaisement, combien elle apprécie d'être en mouvement? Plaisir qu'elle exprime aussi lors des ateliers où le corps est mis en action comme le chant, la danse, la gym... Un corps encore vivant, c'est un corps parlant.

Finalement, la répétition de la syllabe «pa » peut aussi nous évoquer le mot «papa ». Cette interprétation est ici à souligner car quand on l'interroge : «Vous avez dit papa ? elle répond en disant : « maman ».

Lors de la $8^{\text {ème }}$ rencontre, nous passons avec elle devant la porte de sa chambre où sa photo est affichée avec son nom et prénom dessus. Il lui est demandé : «Qui est-ce ? Madame B. sourit en disant «Ah ! Je la connais ». Comme dans la situation du miroir au premier jour, sa propre image fait deux. 
Cette problématique nous amène au «Stade du Miroir » [4] où Lacan met en évidence la fonction de l'imago dans la captation identificatoire à laquelle le sujet est soumis par l'image de l'autre. C'est à ce moment-là que la première identification apparait, avec la formation du $J e:$ l'enfant se reconnaîtra en tant que corps, à partir de l'image de son semblable.

Or, dans les démences, les altérations cognitives affectent les modes d'identification et la représentation de soi. Mais ne pas se reconnaître dans la glace peut nous faire penser à ce qui ne saurait se voir, quelque chose de l'ordre de l'insupportable lié à son image.

Au cours de l'accompagnement de Madame B., le lien transférentiel va se constituer justement comme un miroir, où le fait de se regarder a fonction d'une reconnaissance de son identité corporelle. On n'a jamais vraiment su la place que la psychologue occupait, certainement pas la place de la mère, comme évoqué précédemment, mais était-ce peut-être celle avec qui elle a fait un pas supplémentaire dans le labyrinthe de la vieillesse traversé par la démence.

\section{Madame L. et le rythme de ses pas}

Madame L. attire l'attention par son état de maigreur, ses jambes longues et fines avec des veines très prononcées et gonflées. Elle a un visage mince éclairé par de grands yeux bleus. Elle déambule avec des pas rythmés, en traînant ses pieds au sol et en faisant des pauses. Madame L. va jusqu'à la porte des escaliers, où elle s'arrête quelques secondes avant de la refermer et revenir sur son parcours. Quand on se présente à elle et qu'on lui propose un temps d'échange elle ne répond pas, rentre dans sa chambre et s'allonge dans son lit. C'est alors qu'elle dit spontanément avoir mal au ventre. Il lui est demandé si elle connait la raison de cette douleur. Elle répond : «Elle m'a donné plein de chocolats, j’ai trop mangé ». Malgré les tentatives de conversation par la suite, Madame L. reste allongée avec la main sur le ventre, sans dire un mot. Il lui est évoqué de reporter l'échange à un moment où elle se sentira 
plus disposée. Elle sourit, comme si on avait enfin compris que notre présence n'était pas la bienvenue. Dès qu'elle est laissée seule, Madame L. se relève et repart dans son parcours d'errance habituelle qui peut durer des heures.

Après un temps d'observation, il est noté que cette patiente ne dépasse jamais la porte des escaliers. On ne pourrait pas dire si la porte fait office de point de départ ou d'arrivée, mais elle semble avoir un rôle de limite entre dehors et dedans. En effet, cette porte délimite la topologie de l'espace physique de l'étage et nous ouvre une piste concernant sa position psychique.

Madame L., 94 ans, est arrivée à la résidence en septembre 2016, après un hébergement temporaire de deux mois dans un autre établissement car elle présentait des troubles du comportement importants impliquant un délire de vol et parfois des hallucinations. Selon son dossier médical, il lui est aussi diagnostiqué une maladie d'Alzheimer. Ses troubles auditifs font obstacle à la possibilité d'échange par la parole. L'équipe soignante dispose d'un casque sans fil qu'ils mettent à disposition de certains résidents pour améliorer l'écoute et la communication, surtout au moment d'activités en groupe ou pour les familles.

Lors de la prochaine rencontre, la psychologue se procure cet outil avant de monter à l'étage où se trouve la chambre de Madame L. Elle est dans le petit salon où la radio diffuse du jazz, la salue d'abord et lui propose de mettre le casque, ce qu'elle fait. Elle démarre ses pas aussitôt et la psychologue lui demande si elle peut l'accompagner. Madame L. lève la main, la paume de la main tournée, en faisant un signe de «Stop ». La psychologue lui dit : « Ah avec ce geste, je comprends que vous ne voulez pas que je vous accompagne, c'est bien ça?». Madame L. hoche la tête comme pour dire oui. Ensuite, elle se dirige vers sa chambre et ferme la porte. Quelques secondes après, elle la rouvre et part dans le sens opposé. 
Elle continue son parcours encore une dizaine de minutes, à un pas rythmé et sans dire un mot. Il lui est alors signifié que le casque doit être repris pour être restitué à l'accueil. Etonnée, Madame L. répond en disant : « Non. J'en ai besoin ».

Dans son histoire de vie - document rempli à son entrée à la résidence par sa fille - on apprend que Madame L. n'a jamais eu de profession et qu'elle était une passionnée de musique. En effet elle jouait du violon et donnait des leçons de piano. A l'époque de son entrée en 2016, le psychologue lui avait demandé ce qui l'inquiétait ou ce qui lui faisait peur, Madame L. avait répondu : «d'être enfermée » et ensuite «C'est pénible de devoir quitter la maison ».

Eiguer [5] évoque dans «L'inconscient de la maison» les enjeux psychiques d'un déménagement où «on risque de s'oublier, on devient dissocié, coupé en deux. Déménager, dit-il, ressemble à un retissage de fils psychiques, de liens, plus qu'à un transport d'objets ». Mais, peut-on parler de déménagement lorsqu'un sujet entre en EHPAD ? Tout dépend des conditions d'intégration de chacun, mais surtout du savoir-faire de chaque inconscient face à la séparation, voire la rupture d'un mode de vie. Lorsque les effets de la démence augmentent, certains résidents peuvent exprimer leur souhait de partir, pas forcément en dehors de l'établissement, mais hors d'eux-mêmes [6]. Dans ce sens, pourrait-on considérer l'errance comme un exil de soi-même?

Dans le cas de cette résidente, la présence de l'autre semble perturber son rythme de vie, où l'errance joue un rôle d'apaisement, une sorte de parole qui est incarnée dans son corps. L'intervention des soignants au moment des soins et lors de repas est souvent perçue comme étant une agression. Cet extrait des premiers échanges avec Madame L. nous amène à faire l'hypothèse que pour elle, l'action d'errer est une solution qui lui procure un équilibre précaire mais salutaire pour garder un semblant d'indépendance.

L'accompagnement de Madame L. est encore en cours et les tentatives d'inscrire son errance dans la dimension symbolique échouent. Le travail de construction d'un lien transférentiel est 
confronté à des difficultés du fait de ne plus pouvoir entendre. Cela demande à la psychologue un réaménagement de l'usage de certains dispositifs de médiation, comme par exemple : le casque, la musique, le rythme de ses pas. L'acceptation de Madame L. pour ces outils témoigne qu'elle mène toujours sa danse.

\section{L'accompagnement psychanalytique des sujets errants}

Les manifestations corporelles observées dans la clinique des démences mettent en évidence le lien indissociable entre le somatique et la psyché. Suite à ces deux extraits, nous sommes amenés à nous interroger sur les similarités et les différences entre l'errance de Madame B. et Madame L.

L'activité motrice répétitive semble être, pour les deux, une modalité d'apaisement de l'angoisse, une stratégie d'existence passant par une manifestation qui mobilise le corps. Un corps qui ne cesse pas de s'inscrire dans un parcours qui nous mène à des territoires subjectifs où la parole dérive. En revanche, le lien transférentiel opère différemment dans les deux cas. Accompagner Madame $\mathrm{B}$ dans ses pas a été possible car elle a donné un sens à la présence de l'autre. Alors que pour Madame L, la présence de l'autre est repérée plutôt comme un élément perturbateur dans son errance.

A partir de ce matériel clinique, on peut mettre en évidence la singularité de la clinique de l'errance et la richesse du travail auprès de sujets dits «déments ». La démence, c'est ce devant quoi un analyste ne doit reculer en aucun cas. Nous pouvons nous inspirer de la citation de Lacan [7] évoquant initialement la psychose, mais qui nous sert ici comme direction pour un positionnement co-errant avec la pratique du psychologue en EHPAD.

\section{Considérations finales}


La clinique des démences met en avant le processus de «rabattement de la psyché vers le soma » [8] et l'errance, qui révèle non seulement une activité motrice où la question du corps est mise au premier plan, mais aussi un mode d'expression montrant la persistance de la dimension subjective du sujet dément [9]. Le travail d'accueil et d'écoute des symptômes a pour finalité de maintenir en vie l'inconscient de chaque sujet comme forme de restitution de sa dignité et subjectivité.

Pour finir, dans ces recherches sur le terme d'errance chez Lacan, on s'aperçoit que cette notion n'est pas très développée, vu qu'elle n'est pas un concept psychanalytique. Mais, dans les Ecrits, le terme est fréquemment utilisé, et à chaque fois, il est rapporté à l'analyste. Dès son «Intervention sur le transfert », Lacan nous dit qu'à la fois le transfert est le révélateur des moments d'errance de l'analyste et ce qui permet à l'analyste de s'orienter [10]. Et quelques années plus tard, en 1973, Lacan [11] consacre une année de son enseignement à un séminaire intitulé «Les non-dupes errent ». Voilà une piste précieuse pour soutenir le travail thérapeutique auprès des sujets qui errent. Etre à l'écoute du sujet peu importe son âge ou sa démence est toujours un pari gagnant pour le psychologue. C'est à partir de là que nous pouvons orienter notre clinique, une clinique de l'invention qui met en valeur les effets du réel dans les possibles traitements du sujet, une clinique qui marche surtout au cas par cas, éclairée par la psychanalyse appliquée en institution.

\section{Déclaration de liens d'intérêts}

L'auteur déclare ne pas avoir de liens d'intérêts en relation avec cet article. 


\section{Références}

[1] Strubel D, Corti M. La déambulation chez les patients déments. Psychol NeuroPsychiatr Vieil $2008 ; 6$ (4) : 259-64.

[2] Lacan J. Le Séminaire X, L’angoisse (1962-1963). Paris: Seuil, 2004.

[3]Freud S, Pulsions et destins des pulsions, (1915). Paris : Payot, coll. "Petite Bibliothèque Payot", 2010.

[4] Lacan J. Le stade du miroir (1949). In : Lacan, Ecrits I. Paris: Seuil, 1999.

[5] Eiguer A. L’inconscient de la maison. Paris : Dunod, 2004.

[6] Brossard F, Caron R. Sorties intempestives de personnes atteintes de maladie d'Alzheimer en EHPAD : perspectives thérapeutiques. Encéphale 2016 ; 42(5) : 484-91.

[7] Lacan J. Ouverture de la section clinique. Ornicar ? 1977 ; 1(9) : 7-14.

[8] Grosclaude M. Démence et psychosomatique ? A propos des rapports psyché/soma dans la démence. Psychol Med 1991 ; 23(8) : 917-22.

[9] Grosclaude M. A propos de la persistance de l'humain : du langage et du corps dans la psychothérapie. Rev Fr Psychiatr Psychol Med 1998 ; 1(20) : 38-40.

[10] Lacan J. Intervention sur le transfert (1951). In : Lacan, Ecrits I. Paris : Seuil, 1999.

[11] Lacan J. Séminaire XXI, Les non-dupes errent, 13 novembre 1973. http://www.radiolacan.com/fr/topic/215/2 